\title{
SUBNORMALS IN $C^{*}$-ALGEBRAS
}

\author{
F. H. SZAFRANIEC
}

ABSTRACT. We prove, in a $C^{*}$-algebra set-up, the Bram improvement of Halmos' characterization of subnormals: $(1) \Rightarrow(2)$.

In what is the basic paper on subnormal operators [1], Bram proved that if $S$ is a Hilbert space operator such that

$$
\sum_{i, j=0}^{n}\left(S^{* i} S^{j} f_{i}, f_{j}\right) \geqslant 0, \quad f_{0}, \ldots, f_{n} \in H
$$

then

$$
\sum_{i, j=0}^{n}\left(S^{* i+1} S^{j+1} f_{i}, f_{j}\right) \leqslant\|S\|^{2} \sum_{i, j=0}^{n}\left(S^{* i} S^{j} f_{i}, f_{j}\right), \quad f_{0}, \ldots, f_{n} \in H
$$

This simplifies Halmos' characterization of subnormal operators.

In his recent paper [2] Bunce refers to an element $s$ in a unital $C^{*}$-algebra $A$ as subnormal if

$$
\sum_{i, j=0}^{n} a_{j}^{*} s^{* i} s^{j} a_{i} \geqslant 0, \quad a_{0}, \ldots, a_{n} \in A
$$

It is required for $s$ to satisfy an inequality like (0) that

$$
\sum_{i, j=0}^{n} a_{j}^{*} s^{* i+1} s^{j+1} a_{i} \leqslant\|s\|^{2} \sum_{i, j=0}^{n} a_{j}^{*} s^{* i} s^{j} a_{i}, \quad a_{0}, \ldots, a_{n} \in A
$$

Then (p. 106) he writes "there should be a direct $C^{*}$-algebraic proof that (1) implies (2) without using Theorem 1 of [1] but we have been unable to find such a proof." Here we want to give such a proof adopting arguments of [3] and [4].

Take a positive linear functional $\varphi$ on $A$ and define $\omega(a, b)=\varphi\left(\sum_{i, j} b_{j}^{*} s^{* i} s^{j} a_{i}\right)$ where $a=\left\{a_{i}\right\}, b=\left\{b_{i}\right\}$ are finitely nonzero sequences of elements of $A$. Denote by $X$ the linear space of such sequences. $\omega$ is a positive definite (hermitian) bilinear form on $X \times X$. Thus we have the Schwarz inequality $|\omega(a, b)|^{2} \leqslant \omega(a, a) \omega(b, b)$.

Received by the editors January 23, 1981 and, in revised form, April 17, 1981.

1980 Mathematics Subject Classification. Primary 46L05; Secondary 47C15. 
Denoting the element $\left\{0,0, \ldots, s^{k} a_{0}, s^{k} a_{1}, \ldots\right\}$ (where the first $k$ coordinates are zero) of $X$ by $\tilde{a}$, the Schwarz inequality gives us

$$
\begin{aligned}
\left(\varphi\left(\sum_{i, j=0} a_{j}^{*} s^{* i+k} s^{j+k} a_{i}\right)\right)^{2} & =\left(\varphi\left(\sum_{j=0, i=k} a_{j}^{*} s^{* i} s^{j}\left(s^{k} a_{i-k}\right)\right)\right)^{2} \\
& =|\omega(\tilde{a}, a)|^{2} \leqslant \omega(a, a) \omega(\tilde{a}, \tilde{a}) \\
& =\varphi\left(\sum_{i, j=0} a_{j}^{*} s^{* i} s^{j} a_{i}\right) \varphi\left(\sum_{i, j=0} a_{j}^{*} s^{* i+2 k} s^{j+2 k} a_{i}\right) .
\end{aligned}
$$

Using this and a mathematical induction we have

$$
\left(\varphi\left(\sum_{i, j} a_{j}^{*} s^{* i+1} s^{j+1} a_{i}\right)\right)^{2^{k}} \leqslant\left(\varphi\left(\sum_{i, j} a_{j}^{*} s^{* i} s^{j} a_{i}\right)\right)^{2^{k}-1} \cdot \varphi\left(\sum_{i, j} a_{j}^{*} s^{* i+2^{k}} a^{j+2^{k}} a_{i}\right)
$$

and consequently

$$
\left(\varphi\left(\sum_{i, j} a_{j}^{*} s^{* i+1} s^{j+1} a_{i}\right)\right)^{2^{k}} \leqslant\left(\varphi\left(\sum_{i, j} a_{j}^{*} s^{* i} s^{j} a_{i}\right)\right)^{2^{k}-1}\|\varphi\|\left(\sum_{i}\left\|a_{i}\right\|\left\|s^{i}\right\|\right)^{2}\|s\|^{2^{k+1}} .
$$

Taking the $2^{k}$ th root and passing to the limit we get

$$
\varphi\left(\sum_{i, j=0}^{n} a_{j}^{*} s^{* i+1} s^{j+1} a_{i}\right) \leqslant\|s\|^{2} \varphi\left(\sum_{i, j=0}^{n} a_{j}^{*} s^{* i} s^{j} a_{i}\right) .
$$

Since $\varphi$ is arbitrary, in conclusion, we establish (2).

\section{REFERENCES}

1. J. Bram, Subnormal operators, Duke Math. J. 22 (1955), 75-94.

2. J. W. Bunce, A universal diagram property of minimal normal extensions, Proc. Amer. Math. Soc. 69 (1978), 103-108.

3. F. H. Szafraniec, On the boundedness condition involved in a general dilation theory, Bull. Acad. Polon. Sci. Sér. Sci. Math. Astronom. Phys. 24 (1976), 877-881.

4. __ Dilations on involution semigroups, Proc. Amer. Math. Soc. 66 (1977), 30-32.

UNiWersytet Jagielloński, InStytut Matematyki, Ul. Reymonta 4, 30059 KRakow, Poland 